Article

\title{
Isolation and Structure Characterization of an Antioxidative Glycopeptide from Mycelial Culture Broth of a Medicinal Fungus
}

\author{
Jian-Yong Wu *, Xia Chen and Ka-Chai Siu \\ Department of Applied Biology \& Chemical Technology, State Key Laboratory of Chinese Medicine \\ and Molecular Pharmacology in Shenzhen, The Hong Kong Polytechnic University, Hung Hom, Kowloon, \\ Hong Kong; E-Mails: xiachentiger@gmail.com (X.C.); chai_chai_1987@hotmail.com (K.-C.S.) \\ * Author to whom correspondence should be addressed; E-Mail: jian-yong.wu@polyu.edu.hk; \\ Tel.: +852-3400-8671; Fax: +852-2364-9932.
}

Received: 20 June 2014; in revised form: 1 September 2014 / Accepted: 11 September 2014 / Published: 29 September 2014

\begin{abstract}
A novel glycopeptide (Cs-GP1) with an average molecular weight (Mw) of $6.0 \mathrm{kDa}$ was isolated and purified by column chromatography from the lower $M \mathrm{w}$ fraction of exopolysaccharide (EPS) produced by a medicinal fungus Cordyceps sinensis Cs-HK1. Its carbohydrate moiety was mainly composed of glucose and mannose at 3.2:1.0 mole ratio, indicating an O-linked glycopeptide. The peptide chain contained relatively high mole ratios of aspartic acid, glutamic acid and glycine (3.3-3.5 relative to arginine) but relatively low ratios of tyrosine and histidine. The peptide chain sequence analyzed after trypsin digestion by LC-MS was KNGIFQFGEDCAAGSISHELGGFREFREFLKQAGLE. Cs-GP1 exhibited remarkable antioxidant capacity with a Trolox equivalent antioxidant capacity of $1183.8 \mu \mathrm{mol} / \mathrm{g}$ and a ferric reducing ability of $611.1 \mu \mathrm{mol} \mathrm{Fe}(\mathrm{II}) / \mathrm{g}$, and significant protective effect against $\mathrm{H}_{2} \mathrm{O}_{2}$-induced $\mathrm{PC} 12$ cell injury at a minimum dose of $10 \mu \mathrm{g} / \mathrm{mL}$. This is the first report on the structure and bioactivity of an extracellular glycopeptide from the Cordyceps species.
\end{abstract}

Keywords: Cordyceps sinensis; glycopeptide; structure; antioxidant; cell protection

\section{Introduction}

Polysaccharide and protein (PSP) complexes from edible and medicinal fungi have attracted increasing interest for their notable bioactivities such as immunomodulation, antitumor and antioxidant [1-3]. 
Cordyceps (Ophiocordyceps) sinensis, generally called the Chinese caterpillar fungus, is a well-known medicinal fungus in traditional Chinese medicine with a wide range of health promoting and therapeutic functions [4-6]. Because of the scarcity and high price of natural $C$. sinensis organisms, mycelial fermentation has become a favorable process for mass production of fungal biomass and polysaccharides. Although many previous studies on the bioactive molecules from $C$. sinensis fungi have focused on the polysaccharides either extracted from the fungal mycelia or isolated from the liquid medium, a few have attained proteins and peptides from $C$. sinensis or related species. Wong et al. [7] have recently isolated a peptide called Cordymin with a molecular weight of about $10 \mathrm{kDa}$ from $C$. militaris fruit body with strong antifungal activity and antiproliferative activity. This peptide has also been isolated from the mycelia of a $C$. sinensis fungus and shown significant anti-inflammatory and antioxidant effects in animal models [8]. To the best of our knowledge, there is still no reported study on a bioactive peptide produced as an extracellular product by a Cordyceps fungus.

Cs-HK1 is a C. sinensis fungus isolated from natural fruiting body in our lab and has been applied to mycelial culture and liquid fermentation for production of exopolyssacharide (EPS) [9]. The crude EPS attained by ethanol precipitation from the Cs-HK1 fermentation medium had a protein content of up to $20 \%-25 \%(w / w)$, which was found to contribute more than the carbohydrate content did to the antioxidant effects of EPS [10]. In a recent study [11], the EPS from the Cs-HK1 fermentation medium was roughly fractionated into different ranges of molecular weight $(M \mathrm{w})$ by gradient ethanol precipitation. The lower $M \mathrm{w}$ fraction attained at a higher ethanol volume ratio (2-5) had a higher protein content and stronger antioxidant activity. In a later study [12], the low $M \mathrm{w}$ EPS was further fractionated into more pure fractions of PSPs, some of which showed notable antioxidant activities.

The present study was aimed at the purification and structural characterization of an antioxidative glycopeptide from the low $M \mathrm{w}$ EPS fraction produced by the Cs-HK1 fungus in mycelial liquid culture, and evaluation of its antioxidant property through chemical and cell culture assays.

\section{Results and Discussion}

\subsection{Isolation and Molecular Profiles of Cs-GP1 from EPS-2}

EPS-2 was fractionated into five fractions (OF-I, II, III, IV and V) by gel filtration through the Superdex 75 column with RI detection. OF-IV and OF-V had much higher protein contents $(30 \%-50 \%)$ than the other three fractions (6\% or lower) [12]. Fraction OF-V as well as fraction OF-IV (but not OF-I, II and III) also showed the protein absorption peak on UV at $280 \mathrm{~nm}$. Because of its remarkable antioxidant activity, OF-V was further purified by ion exchange chromatography on the DEAE column (Figure 1a), yielding the glycopeptide fraction Cs-GP1. Cs-GP1 had a protein content of 52.3\% (Table 1) and a carbohydrate content of $30 \%$ (determined by phenol-sulfuric method, data not shown), and was recognized as a glycopeptide. 
Figure 1. Fractionation and detection of glycopeptide Cs-GP1: (a) Ion exchange chromatograph purification of Cs-GP1 fraction on a DEAE 52 column (UV detection); (b) HPGPC profile of Cs-GP1 detected by on an Ultrahydrogel 250 column; (c) SDS-PAGE.
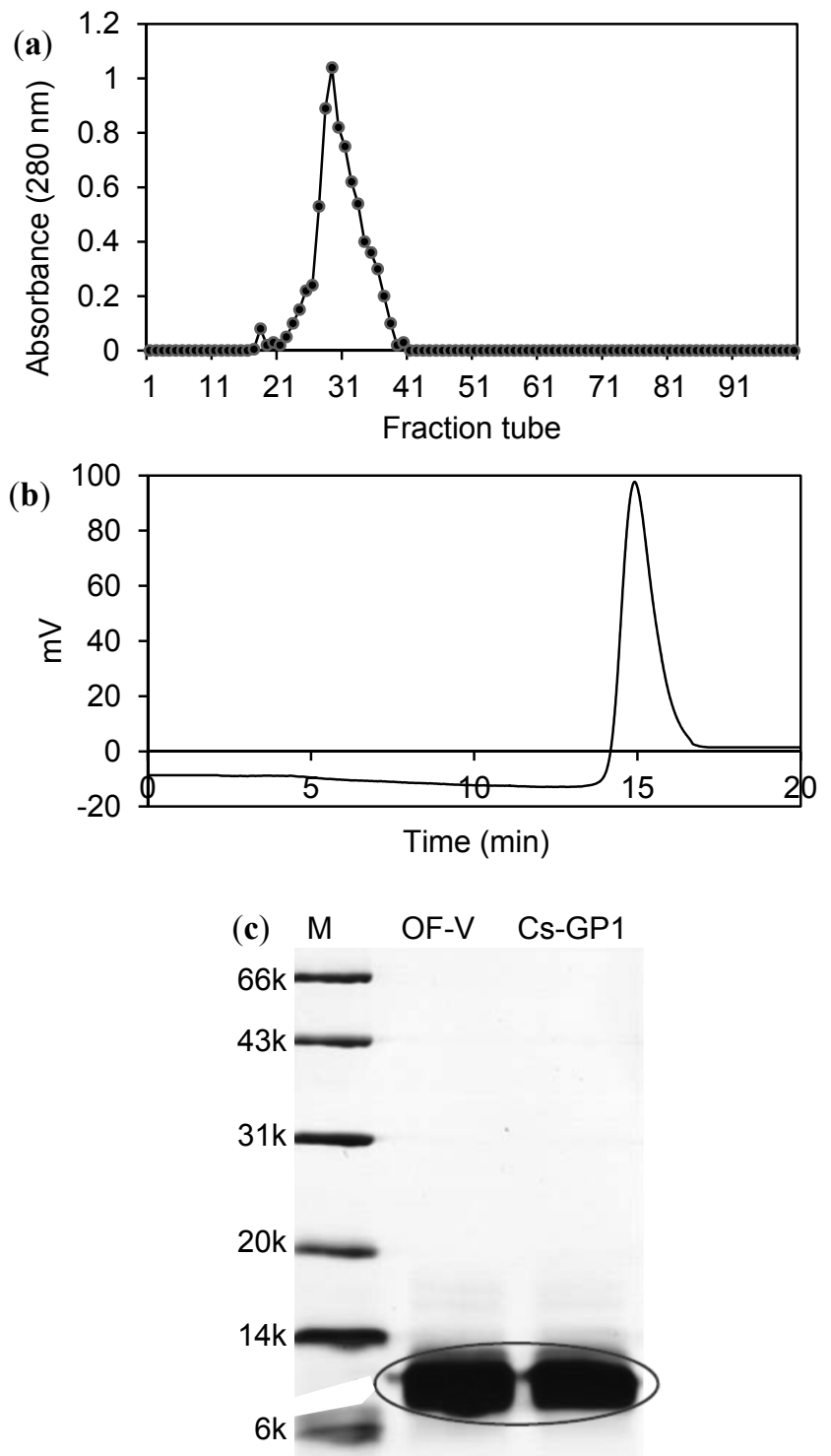

Table 1. Composition and molecular weight of EPS-2 fractions obtained by gel filtration chromatography.

\begin{tabular}{cccccccc}
\hline \multirow{2}{*}{ Fraction } & \multirow{2}{*}{ Protein Content (\%) } & \multirow{2}{*}{ Mw (kDa) } & \multicolumn{5}{c}{ Mole Ratio } \\
\cline { 4 - 8 } & & & Man & Glc & GlcA & Gal & GalN \\
\hline OF-IV & 30.1 & 13 & -- & 0.1 & -- & -- & 2.1 \\
OF-V & 50.5 & 6.0 & 1.0 & 3.2 & 1.5 & 1.0 & 0.96 \\
Cs-GP1 & 52.5 & 6.0 & 1.0 & 3.2 & -- & 0.2 & 0.3 \\
\hline
\end{tabular}

“--": Undetectable.

The Cs-GP1 fraction showed a single peak on HPGPC (Figure 1b), which was calibrated to an average $M \mathrm{w}$ of $6.0 \mathrm{kDa}$ (Table 1). It exhibited a single thick band on SDS-PAGE (Figure 1c), corresponding to $8.0 \mathrm{kDa} M \mathrm{w}$. The MALDI-TOF-MS spectrum of Cs-GP1 (Supplemental data Figure 1) 
revealed a major peak at $6057 \mathrm{~m} / \mathrm{z}$ and two small fragments at 4423 and $1634 \mathrm{~m} / \mathrm{z}$, which were probably derived from the hydrolysis of the fragment at $6057 \mathrm{~m} / z$ during ionization. The fragment with $\mathrm{m} / \mathrm{z}$ at 1634 was most likely the glyco-chain, whereas the $m / z$ at 4423 was the peptide chain. These analytical results all indicated the molecular homogeneity of Cs-GP1, suitable for further structure analysis.

\subsection{Sugar and Amino Acid Constituents of Cs-GP1}

Monosaccharide analysis indicated that Cs-GP1 was composed mainly of glucose (Glc) and mannose (Man) at 3:1 mole ratio and a small proportion of GalN and Gal (Table 1) (Supplemental data: Figure 2 HPLC profile of Cs-GP1). Furthermore, the high contents of Glc and Man are indicative of an O-linked glyco-chain. As shown by the amino acid analysis (Table 2), Cs-GP1 had high mass contents of glutamic acid (Glu), aspartic acid (Asp), glycine (Gly) and cysteine (Cys) (76.6-40.6 $\mu \mathrm{g} / \mathrm{mg}$ ), but relatively low contents of threonine (Thr), tyrosine (Tyr) and histidine (His).

Figure 2. IR and NMR spectra of Cs-GP1: (a) IR; (b) ${ }^{1} \mathrm{H}$ NMR.

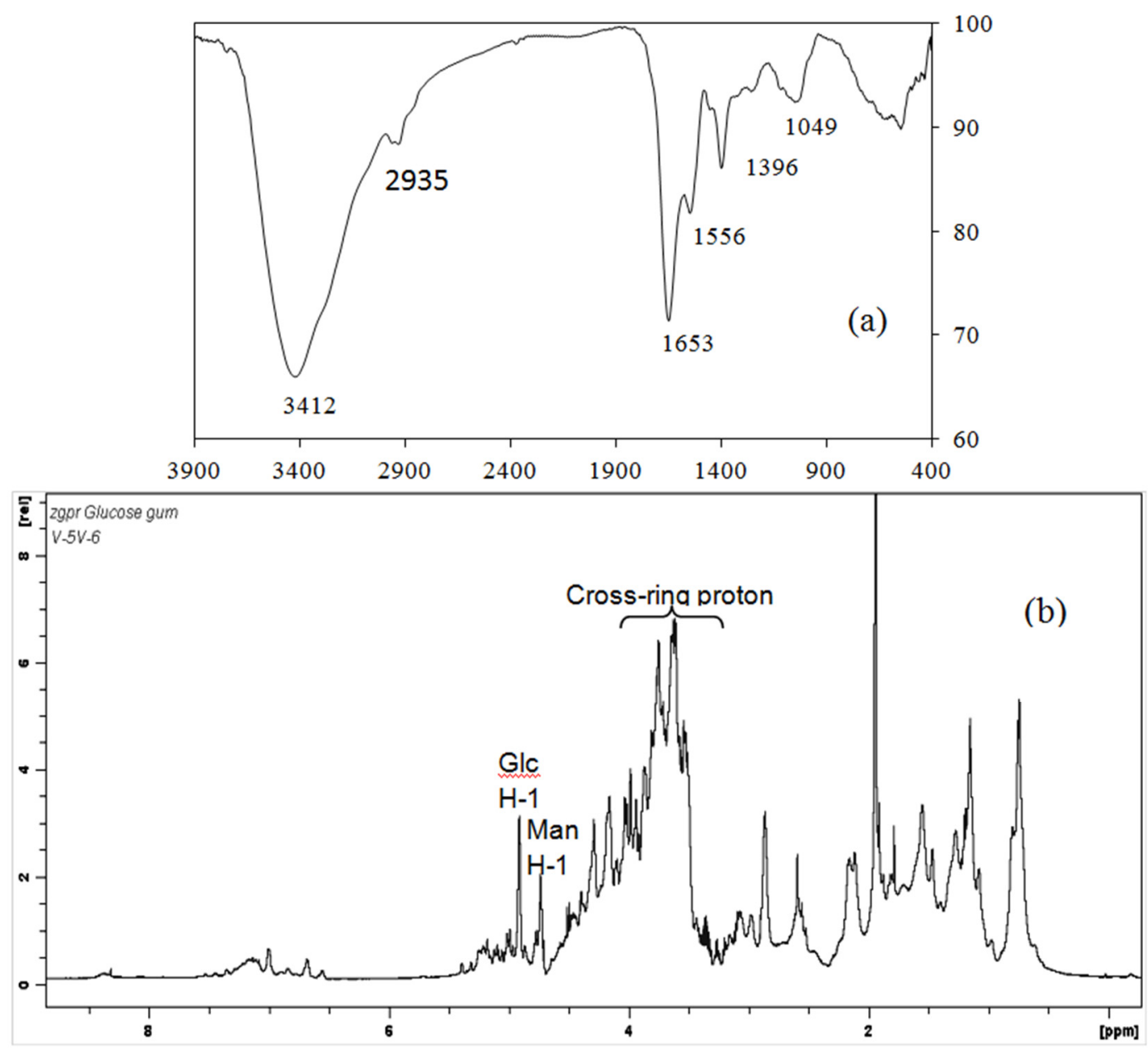


Table 2. Amino acid composition of Cs-GP1.

\begin{tabular}{lcccc}
\hline \multicolumn{2}{c}{ Amino Acid } & Content $(\boldsymbol{\mu g} / \mathbf{m g}) *$ & Mole Ratio $* *$ & $\boldsymbol{M}_{\mathbf{W}}$ \\
\hline 1 & Asp & $66.89 \pm 1.23$ & 3.28 & 133 \\
2 & Thr & $9.76 \pm 0.31$ & 0.54 & 119 \\
3 & Ser & $33.56 \pm 1.11$ & 2.09 & 105 \\
4 & Glu & $76.61 \pm 2.41$ & 3.40 & 147 \\
5 & Gly & $40.61 \pm 1.48$ & 3.53 & 75 \\
6 & Ala & $26.27 \pm 0.93$ & 1.93 & 89 \\
7 & Cys & $40.61 \pm 1.56$ & 1.10 & 240 \\
8 & Val & $7.86 \pm 0.36$ & 0.44 & 149 \\
9 & Ile & $5.91 \pm 0.44$ & 0.29 & 131 \\
10 & Leu & $7.12 \pm 0.28$ & 0.35 & 131 \\
11 & Tyr & $3.96 \pm 0.19$ & 0.14 & 181 \\
12 & Phe & $30.52 \pm 1.13$ & 1.20 & 165 \\
13 & Lys & $30.22 \pm 0.79$ & 1.35 & 146 \\
14 & His & $11.42 \pm 0.52$ & 0.48 & 155 \\
15 & Arg & $26.61 \pm 0.72$ & 1.00 & 174 \\
16 & Pro & $19.59 \pm 0.67$ & 1.11 & 115 \\
\hline Total amino acid & $437.52 \pm 16.15$ & &
\end{tabular}

Total amino acid $437.52 \pm 16.15$

* Mean \pm standard deviation (SD) of triplicate measurements; ** Mole ratio relative to Arg.

\subsection{IR and NMR Spectra}

Figure 2a shows the FT-IR spectrum of Cs-GP1. The peaks at 3407 and $2935 \mathrm{~cm}^{-1}$ are characteristic of $\mathrm{O}-\mathrm{H}$ and $\mathrm{C}-\mathrm{H}$ stretching vibration from the glyco-chain and amino acid, respectively. Between 1800 and $400 \mathrm{~cm}^{-1}$ are the characteristic bands of amino acids, i.e., the peak at $1650.9 \mathrm{~cm}^{-1}$ assigned to amide I band from the peptide, the peak at $1560.7 \mathrm{~cm}^{-1}$ to amide group II vibration [13], and the peak at $1396.0 \mathrm{~cm}^{-1}$ to high content of COO-, which was probably from Asp and Glu [14]. The peak at $1054 \mathrm{~cm}^{-1}$ is attributed to $\mathrm{C}-\mathrm{O}-\mathrm{C}$ stretching vibration, and $846 \mathrm{~cm}^{-1}$ to $\alpha$ conformation in the sugar units. The molecular structure and composition deduced from IR are consistent with the results of monosaccharide and amino acid analysis (Tables 1 and 2).

As for the ${ }^{1} \mathrm{H}$ NMR spectrum (Figure $2 \mathrm{~b}$ ), the peaks between 8.1 and $8.5 \mathrm{ppm}$ are assigned as reported previously $[15,16]$ to the $\beta-\mathrm{NH}$ signals of amino acid, those between 6.5 and $7.5 \mathrm{ppm}$ are assigned to $\alpha-\mathrm{NH}$ signals, and between 4.8 and $5.5 \mathrm{ppm}$ assigned to the anomeric signals of the sugar units. These peaks between 3.5 and $4.7 \mathrm{ppm}$ are assigned to the $\mathrm{C}-\mathrm{H}$ signals of both amino acids and sugar units, and those between 1.8 and 2.5 and 0.5 and 1.8 assigned to $\gamma$ and $\lambda \mathrm{C}-\mathrm{H}$ signals of the amino acid. The relatively strong signal between 4.0 and 4.6 and 3.6 and 3.9 may be attributed to the high contents of Gly, Ala and Asp; the peaks between 2.0 and 2.5 may be attributed to the $\mathrm{H}$ signals in Glu and Asp. These results are consistent with the above amino acid composition (Table 2).

\subsection{Amino Acid Sequence of Peptide Chain}

After extensive in-gel trypsin digestion, Cs-GP1 was degraded into peptide fragments with $\mathrm{m} / \mathrm{z}$ values ranging from 700-2800 (Figure 3a). Table 3 shows the de novo sequences of the peptide 
fragments detected by LC-ESI-MS-MS. The overall peptide sequence was derived from the overlapped sequences among the fragments as follows, GKNGIFQFGEDCAAGSLSEHLGGFREFREFLKAGNLE. The total mass (4102) was $321 \mathrm{~m} / z$ lower than the mass (4423) of the native Cs-GP1 derived from MALDI-TOF-MS, which was probably attributed to a small amount of peptide residual retained in the glyco-chain after enzymatic digestion. Based on the results from the sequence analysis and the above monosaccharide analysis, we suggest that the glyco-chain was attached to serine (Ser) in the peptide chain by O-linkage. The peptide chain sequence contained relatively large number of Ala (3), Gly (6) and Glu (5), which was consistent with the high contents found from amino acid composition analysis.

Figure 3. A peptide sequence from trypsin-digested Cs-GP1 detected by MALDI-TOF-MS/MS: (a) Spectrum of the peptide sequence; (b) Spectrum of its fragment with $m / z$ at 2470 .
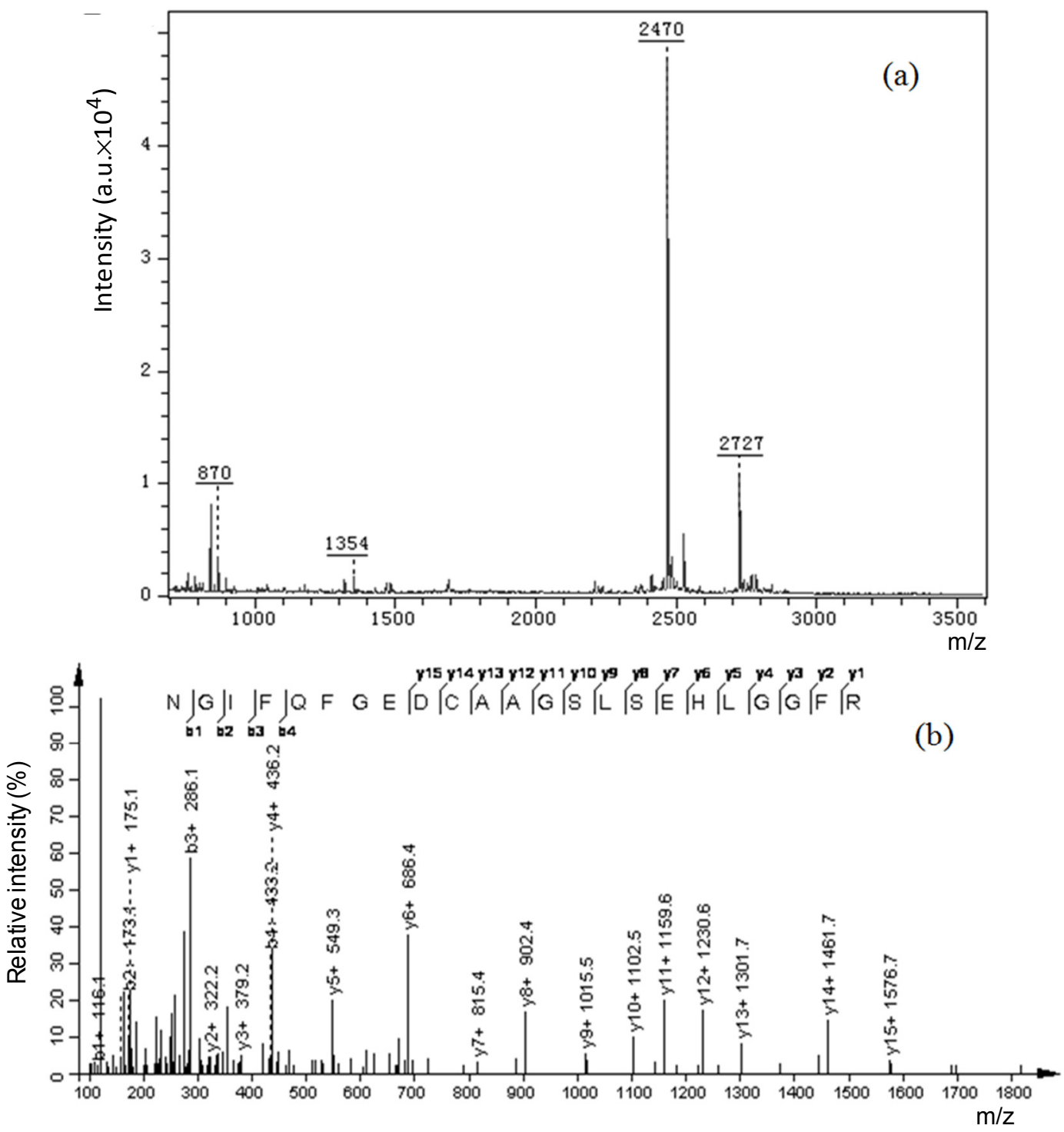
Table 3. De novo peptide sequences detected by ESI-MS/MS of trypsin digestion products of Cs-GP1.

\begin{tabular}{ccc}
\hline Fragments & Mass & Sequence \\
\hline A & 1758 & DCAAGSLSEHLGGFRE * \\
B & 1412 & AAGSLSEHLGGFR \\
C & 1359 & AGSLSEHLGGFR \\
D & 2470 & NGIFQFGEDCAAGSLSEHLGGFR \\
E & 1930 & KNGIFQFGEDCAAGSLSE \\
F & 1987 & GKNGIFQFGEDCAAGSLSE \\
G & 2172 & HLGGFREFLKAGNLE \\
Whole chain & 4102 & GKNGIFQFGEDCAAGSLSEHLGGFREFREFLKAGNLE \\
\hline
\end{tabular}

* Detected by both MALDI-TOF/MS/MS and LC-MS-MS ( $\left.{ }^{\mathrm{E} E S}\right)$.

The peptide chain sequence was further confirmed by MALDI-TOF-MS-MS analysis of the main peptide fragment with $\mathrm{m} / \mathrm{z}$ at 2470 (Figure 3b). The fragment sequence was identified as NGIFQFGEDCAAGSLSEHLGGFR, which matched closely with the peptide chain sequence derived from LC-ES-MS-MS.

\subsection{Antioxidant Activities}

Figure $4 \mathrm{a}$ shows the scavenging (or inhibiting) effect on $\mathrm{ABTS}^{-+}$radicals and Figure $4 \mathrm{~b}$ the ferric reducing power of Cs-GP1, both exhibiting a linear correlation with concentration. From these activity versus concentration curves, the following activity indexes were derived: $\mathrm{IC}_{50}$ of $35 \mu \mathrm{g} / \mathrm{mL}$ for inhibition of $\mathrm{ABTS}^{*+}$ radicals, TEAC value of $1180 \mu \mathrm{mol}$ Trolox/g, and FRAP value of $610 \mu \mathrm{mol}$ $\mathrm{Fe}(\mathrm{II}) / \mathrm{g}$. The activity indexes for fraction IV derived from these two assays were $\mathrm{IC}_{50} 0.19 \mu \mathrm{g} / \mathrm{mL}$ on $\mathrm{ABTS}^{\cdot+}$ radicals, $360 \mu \mathrm{mol}$ Trolox/g and $43 \mu \mathrm{mol} \mathrm{Fe}$ (II)/g. In comparing these antioxidant activity indexes, Cs-GP1 had a much higher antioxidant capacity than OF-IV and the other three fractions (OF-I,II,III) which were composed mainly of carbohydrate as reported previously [12]. The strong antioxidant capacity of Cs-GP1 was also confirmed by the cell culture test (Figure 4c), showing a dose-dependent protecting effect against $\mathrm{H}_{2} \mathrm{O}_{2}$-induced cell viability loss of $\mathrm{PC} 12$ cells. The protective effect was statistically significant at $p<0.05$ in the concentration range of $10-200 \mu \mathrm{g} / \mathrm{mL}$ and at $200 \mu \mathrm{g} / \mathrm{mL}$ maintained a cell viability of $63 \%$ after exposure to $\mathrm{H}_{2} \mathrm{O}_{2}$.

Figure 4. Antioxidant activities of Cs-GP1: (a) Scavenging (eliminating) ABTS $^{\cdot+}$ radicals; (b) Ferric reducing power (FRAP); (c) Protecting against $\mathrm{H}_{2} \mathrm{O}_{2}$-inducd viability loss of PC12 cells (exposed to $80 \mu \mathrm{M} \mathrm{H}_{2} \mathrm{O}_{2}$ for $1 \mathrm{~h}$; * significant effect at $p<0.05$ ).

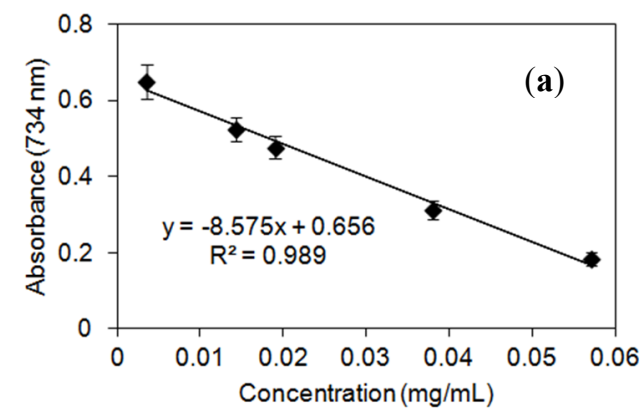


Figure 4. Cont.
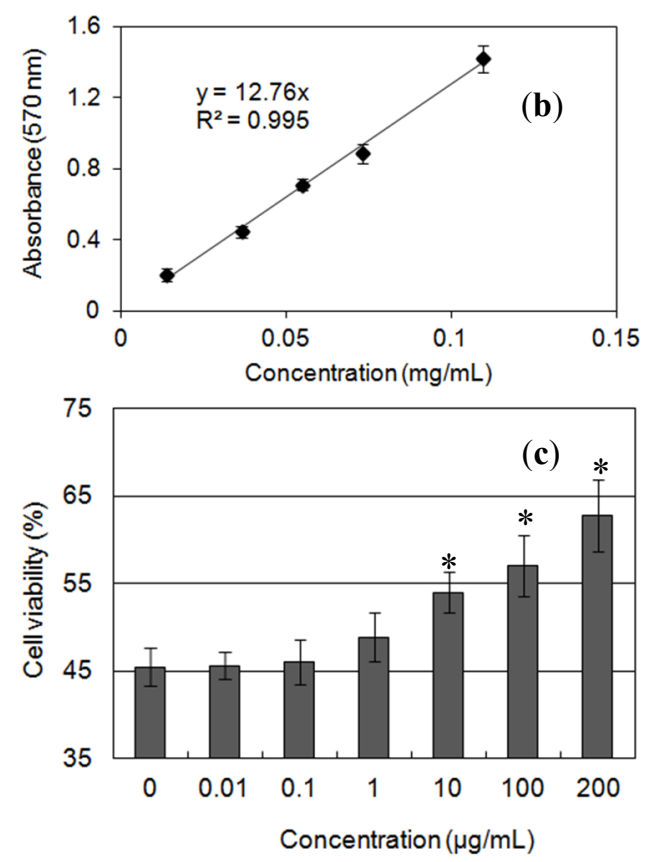

\subsection{Discussion}

Glycosylation is one of the most common posttranslational modifications of proteins in eukaryotic organisms and has significant influence on protein folding and intracellular trafficking [17,18]. The oligosaccharide moieties of glycoproteins are covalently bonded to the proteins in N- or O-linked form. Glycoproteins are involved in many important cellular communication processes associated with cell adhesion, host-pathogen interaction, and immune responses [19-23]. Therefore, the isolation and characterization of homogeneous glycoproteins and glycopeptides are needed for investigation of the biological functions and the structure-activity relationships.

Cs-GP1 isolated and fractionated from the low Mw EPS of Cs-HK1 fungus has been identified as an O-linked glycopeptide with relatively high contents of Glc and Man in the oligosaccharide portion and its peptide portion contained high contents of Ala, Gly and Asp amino acids. Cs-GP1 showed strong antioxidant activity in both chemical and cell culture assays. There is ample literature on the strong antioxidant properties of naturally-occurring peptides or produced by hydrolysis of food proteins from plants and animals [3]. Most of the antioxidant food peptides are in the $M \mathrm{w}$ range of 500-1800 Da. The bioactivities as well as the properties of peptides are dependent on the amino acid composition and sequence. As free amino acids are not active in general, the amino acid sequences are crucial for the antioxidative activity of peptides. Some previous studies have suggested that high contents of some amino acid species such as Asp, Gly and Ala were significant factors for several antioxidative peptides from soybeans [24] and jumbo squids [25]. However, no general rule of thumbs has been established for the active amino acid composition and sequences. 


\section{Experimental Section}

\subsection{Materials}

The Cs-HK1 fungus used in this study was previously isolated in our lab from the fruiting body of a wild C. sinensis organism [10] and has been preserved at the China General Microbiological Culture Collection Center (Registration No. 6004). Ultrahydrogel 250 columns were acquired from Waters Corp. (Milford, MA), DEAE-cellulose anion-exchange resin from Whatman (Brentford, UK), papain and cysteine from Fluka (Seelze, Germany). The protein $M \mathrm{w}$ markers of 6-66 kDa for SDS-PAGE were from GE Healthcare. The carbohydrate standards, D-mannose, L-fucose, L-arabinose, D-galacturonic acid and lactose were from Sigma (St. Louis, MO, USA), the derivatization reagent, 1-phenyl-3-methyl-5-pyrazolone (PMP) from Sinopharm Chemical Reagent Co., Ltd. (Shanghai, China), and the $\mathrm{D}_{2} \mathrm{O}$ (99.8\%) for NMR from Cambridge Isotope Laboratories Inc. (Andover, MA, USA). Sequencing grade modified trypsin was from Promega (Madison, WI, USA), alpha-cyano-4-hydroxy-cinnamic acid (CHCA) and trifluoroacetic acid (TFA) were from Sigma, and formic acid (FA) and ACN from Merck (Darmstadt, Germany).

Trolox ([(S)-(2)-6-hydroxy-2,5,7,8-tetramethyl-chroman-2-carboxylic acid]) and 2,2'-azinobis (3-ethylbenzothiazoline-6-sulfonic acid) (ABTS) for the antioxidant assays were purchased from Calbiochem/EMD (Gibbstown, NJ, USA). The PC12 cell line for the cell culture tests was obtained from American Type Culture Collection, the RPMI 1640 medium, and fetal bovine serum from Gibco-BRL (Grand Island, NY, USA), $\mathrm{H}_{2} \mathrm{O}_{2}$ and MTT (3-(4,5-dimethylthiazol-2-yl)-2, 5-diphenyltetrazolium bromide) from Sigma.

\subsection{Isolation and Purification of EPS from Cs-HK1 Mycelial Culture}

Figure 5 illustrates the procedure for isolation and fractionation of EPS from the Cs-HK1 mycelial culture, and purification of the glycoprotein. The Cs-HK1 fungus was cultivated in $250 \mathrm{~mL}$ Erlenmeyer flasks each containing $50 \mathrm{~mL}$ of a liquid medium, shaken constantly at $150 \mathrm{rpm}$ and $20{ }^{\circ} \mathrm{C}$ for 7 days [10]. The mycelial broth was then centrifuged and the supernatant liquid medium was collected for isolation of EPS by ethanol precipitation. The ethanol precipitation was performed in two steps, using 2-volume ratio of ethanol ( $96 \%$ grade) to the liquid medium in the first step to precipitate the high- $M \mathrm{w}$ EPS, followed by another 3-volumes of ethanol in the second step to precipitate the remaining low- $M \mathrm{w}$ EPS. The precipitate was washed with acetone, redissolved in water and lyophilized. EPS-2 ( 0.3 g) was redissolved in $2 \mathrm{~mL}$ distilled water and loaded onto a Superdex 75 gel filtration column $(2.6 \times 60 \mathrm{~cm})$, eluted with $0.3 \mathrm{M} \mathrm{NH}_{4} \mathrm{HCO}_{3}$ at a flow rate of $0.3 \mathrm{~mL} / \mathrm{min}$, and monitored by RI. The peak fractions collected were scanned by UV and the fraction (OF-V) exhibiting an absorption peak was collected as the glycopeptide fraction for the following experiments.

The OF-V fraction was dialyzed against distilled water and lyophilized. It was $(\sim 100 \mathrm{mg})$ then fractionated by anion-exchange chromatography on a DEAE-cellulose column $(2.6 \times 40 \mathrm{~cm})$, eluted with $\mathrm{NaCl}$ on a linear gradient from 0 to $1.0 \mathrm{M}$ (in $0.1 \mathrm{M}$ sodium acetate solution at $\mathrm{pH}$ 5.0) for $500 \mathrm{~min}$ at $1.0 \mathrm{~mL} / \mathrm{min}$, and monitored with $\mathrm{UV}$ at $280 \mathrm{~nm}$. The peak fraction was collected and dialyzed against distilled water and lyophilized, yielding the final glycopeptide Cs-GP1. 
Figure 5. Procedure for isolation and fractionation of EPS from Cs-HK1 mycelium liquid medium and the purification of glycopeptide Cs-GP1.

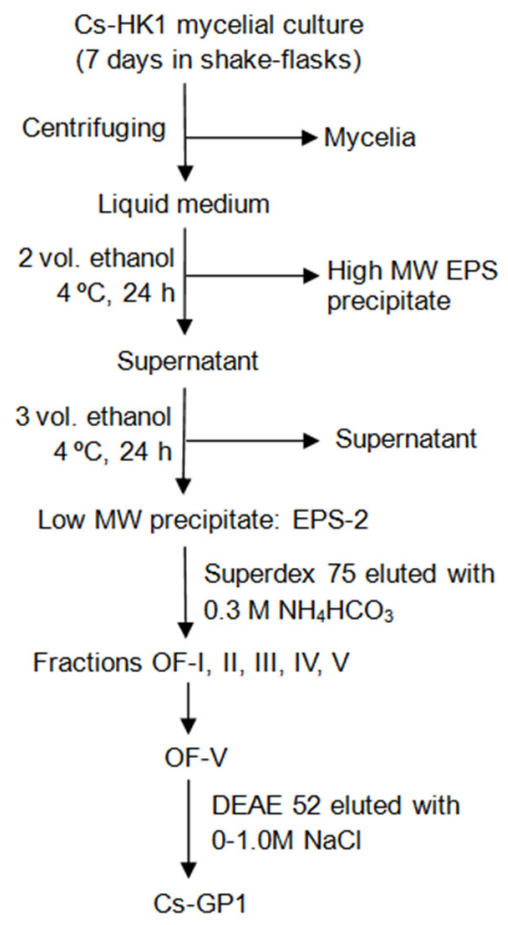

\subsection{Analysis of Cs-GP1 Molecular Composition and Properties}

\subsubsection{Monosaccharide, Amino Acid and Protein Contents}

Monosaccharide composition was analyzed by HPLC as described by Chen et al. [16]. In brief, Cs-GP1 ( 2 mg) was hydrolyzed with $2 \mathrm{M}$ TFA at $110^{\circ} \mathrm{C}$ in nitrogen atmosphere for $8 \mathrm{~h}$, with lactose added as an internal standard. The hydrolysate was dried under vacuum, and then derivatized with $450 \mu \mathrm{L}$ 1-phenyl-3-methyl-5-pyrazolone (PMP) solution (0.5 M, in methanol) and $450 \mu \mathrm{L}$ of $0.3 \mathrm{M} \mathrm{NaOH}$ at $70{ }^{\circ} \mathrm{C}$ for $30 \mathrm{~min}$. The reaction was stopped by neutralization with $450 \mu \mathrm{L}$ of $0.3 \mathrm{M} \mathrm{HCl}$, followed with chloroform extraction ( $1 \mathrm{~mL}$, three times). The extract solution was analyzed by HPLC on a Waters 2870 instrument with an Agilent ZORBAX Eclipse XDB-C18 column $(5 \mu \mathrm{m}, 4.6 \times 150 \mathrm{~mm})$ at $25{ }^{\circ} \mathrm{C}$ with $\mathrm{UV}$ detection at $250 \mathrm{~nm}$. The mobile phase was composed of $0.05 \mathrm{M} \mathrm{KH}_{2} \mathrm{PO}_{4}(\mathrm{pH} 6.9)$ with $15 \%$ acetonitrile (solvent A) and 40\% acetonitrile (solvent B) in water on a gradient from 8\%-19\% B in 25 min.

The amino acid composition was analyzed after hydrolysis of Cs-GP1 (with $6 \mathrm{M} \mathrm{HCl}$ under reduced pressure at $110{ }^{\circ} \mathrm{C}$ for $24 \mathrm{~h}$ ) using a Hitachi 835-50 Amino Acid Analyzer (Hitachi, Tokyo, Japan). The protein content was determined by Lowry method using bovine serum albumin as a standard [26].

\subsubsection{Average Molecular Weight}

The average $M \mathrm{w}$ of Cs-GP1 was analyzed by high pressure gel permeation chromatography (HPGPC) on a Waters instruments (Waters 1515 isocratic pump +2414 refractive index (RI) detector) and calibrated with dextran $M \mathrm{w}$ standards, as reported previously [11,12]. The $M \mathrm{w}$ distribution was also detected by SDS-PAGE (using 4\% stacking gel and 12\% separating gel) and staining with Coomassie Brilliant Blue in comparison with those of protein $M \mathrm{w}$ markers of 6-66 kDa. The Cs-GP1 
sample was dissolved at $1 \mathrm{mg} / \mathrm{mL}$ in distilled water and added at 1:3 volume ratio into a buffer solution of $0.5 \%$ SDS with $1 \%$-mercaptoethanol, and then heated to boiling for $5 \mathrm{~min}$. The gels were stained with Coomassie Brilliant Blue R-250 to visualize proteins.

The molecular weight was analyzed more accurately by the matrix-assisted laser desorption ionization time-of-flight mass spectrometry (MALDI-TOF MS) on an ultrafleXtreme (Brukers, Germany), using CHCA as matrix. The Cs-GP1 sample solution was mixed with 1 volume of matrix solution $(20 \mathrm{mg} / \mathrm{mL}$ of CHCA in acetonitrile/water, 50:50, $v / v)$ in a final concentration of $50 \mu \mathrm{g} / \mathrm{mL}$. Finally, $0.5 \mu \mathrm{L}$ of the mixture was deposited onto the MALDI target plate. All spectra were the results of signal averaging of 200 shots. The instrument was operated at laser energy $20 \%$ (coarse) and $60 \%$ (fine), and resolution 1000 .

\subsubsection{NMR and IR Spectroscopy}

NMR and IR spectroscopy were performed with the same procedures and instruments as described previously [12,15]. In brief, ${ }^{1} \mathrm{H}$ NMR was performed at $600 \mathrm{MHz}$ and ${ }^{13} \mathrm{C} \mathrm{NMR}$ at $150 \mathrm{MHz}$ at room temperature on a Bruker AVANCE III 600 spectrometer with Topspin 3.0 software for data processing. The Cs-GP1 sample ( $30 \mathrm{mg}$ ) was lyophilized with $500 \mu \mathrm{L}$ D2O (99.8\%) twice and then dissolved in $500 \mu \mathrm{L}$ high quality D2O (99.96\%) containing $0.1 \mu \mathrm{L}$ acetone as an internal standard for the ${ }^{1} \mathrm{H}$ chemical shifts. Infrared (IR) spectrum was recorded on a Perkin-Elmer 1600 instrument at room temperature in wave number range of $4000-400 \mathrm{~cm}^{-1}$.

\subsection{Analysis of Peptide Chain Sequence}

\subsubsection{In-Gel Digestion}

SDS-PAGE was performed as described in Section 3.3.2. The gel was washed with Milli-Q water and then stained with $50 \mathrm{~mL}$ of Coomassie staining solution containing $45 \%(v / v)$ methanol, $10 \%(v / v)$ acetic acid, and $0.15 \%(w / v)$ Coomassie Brilliant Blue R350 for $1 \mathrm{~h}$, then finally de-stained for $1-1.5 \mathrm{~h}$ using $100 \mathrm{~mL}$ of destaining solution containing $40 \%(v / v)$ methanol and $10 \%(v / v)$ acetic acid in Milli-Q water. The gel stained by Coomassie was sliced into four pieces of gel matrix, and then cut into about $1 \mathrm{~mm}$ cubes using a razor blade on a clean glass surface. The cubes were transferred into Eppendorf tubes for in-gel trypsin digestion. The in-gel trypsin digestion and the extraction of the tryptic fragments were performed according to well-established protocols [27]. In brief, acetonitrile (ACN) $(25-35 \mu \mathrm{L})$ was added to each tube to dehydrate and shrink the gel pieces. After drying with Speed-VAc, the gel pieces were incubated in $25-35 \mu \mathrm{L}$ of digestion buffer $(0.1 \mu \mathrm{g} / \mu \mathrm{L}$ sequencing grade modified trypsin in $50 \mathrm{mM} \mathrm{NH} \mathrm{HCO}_{3}$ ) for $45 \mathrm{~min}$ in an ice water bath. The mixture was centrifuged at $10,000 \times g$ and the supernatant extract was collected. The remaining gel pieces were incubated in $20 \mu \mathrm{L}$ of $20 \mathrm{mM} \mathrm{NH}_{4} \mathrm{HCO}_{3}$ for $10 \mathrm{~min}$ and the supernatant was collected and combined with previous extract. The remaining gel pieces were extracted with $20 \mu \mathrm{L}$ extraction buffer (50\% ACN, 5\% formic acid) for $20 \mathrm{~min}$ and the extract was combined with the previous ones. Finally, the combined extract was dried by Speed-Vac and stored at $-80{ }^{\circ} \mathrm{C}$ until MS analysis. The tryptic digestion product of Cs-GP1 was subject to MALDI-TOF analysis as described in Section 2.3.2 to 
monitor the degree of degradation and then applied to LC-MS/MS and MALDI-TOF-MS-MS for analysis of the peptide sequences.

\subsubsection{Mass Spectrometry}

For the LC-MS/MS analysis, the digested product samples were desalted using the ZipTip-C18 (Millipore) treatment. The samples were then loaded onto an analytical column $(15 \mathrm{~cm} \times 75 \mu \mathrm{m}$ i.d.; Acclaim@PepMap100 C18, Dionex, Sunnyvale, CA, USA). The nano-flow was eluted at a flow rate of $300 \mathrm{~nL} / \mathrm{min}$ with solvent A (2\% ACN with $0.1 \%$ formic acid) and solvent B (95\% ACN with $0.1 \%$ formic acid). LC analysis was performed on a 40 min staged gradient elution program, $0-4 \mathrm{~min}$ (5\% B), 4-40 $\min (5 \%-35 \%$ B). The column outlet was coupled directly to a high voltage ESI source, which was interfaced to a Shimadzu UFLC-LTQ-Orbitrap HCD, operated at $1.7 \mathrm{kV}$ spray voltage in the nES-LC-MS/MS mode in 200-2500 Da $\mathrm{m} / \mathrm{z}$ range.

The peptide de novo sequences were derived by matching the acquired data with the National Center for Biotechnology Information (NCBI) non-redundant protein database (fungi) using the MASCOT software package (Version 2.3, Matrix Science, London, UK). The peptide mass and MS/MS tolerance were both $0.2 \mathrm{Da}$. The peptides have the allowance of one tryptic missed cleavage, one fixed modification with carb-amidomethyl $(\mathrm{C})$ and one variable modification by oxidation.

MALDI-TOF-MS-MS analysis was performed as described in Section 2.3.2. The trypsin digested peptide was subjected to MS/MS analysis and the results were used to confirm the data from LC-ES-MS-MS.

\subsection{Antioxidant Activity Assays}

The antioxidant activities of Cs-GP1 as well as other EPS fractions attained from were evaluated using two chemical assays, the Trolox equivalent antioxidant capacity (TEAC) and the ferric reducing ability of plasma (FRAP) assay, and a cyto-protection test using $\mathrm{H}_{2} \mathrm{O}_{2}$-induced cell injury, as described previously [11]. In brief, the TEAC assay measures the ability of a compound to eliminate or scavenge $\mathrm{ABTS}^{\circ+}$ radicals using Trolox as a response reference [28]. The EPS sample solution in water was reacted with the $\mathrm{ABTS}^{\circ+}$ solution for $20 \mathrm{~min}$ at room temperature, followed by measurement of the absorbance at $734 \mathrm{~nm}$. The scavenging activity of a sample was correlated with the absorbance decrease, and converted to a TEAC value in $\mu \mathrm{mol}$ Trolox/g sample by calibration with Trolex from 0-30 $\mu \mathrm{M}$. The FRAP assay was performed according to Benzie and Strain [29]. The FRAP reagent was reacted with the EPS sample for $15 \mathrm{~min}$ at room temperature, followed by measurement of absorbance at $593 \mathrm{~nm}$. The reducing power of a sample was correlated to the absorbance increase, and converted to a FRAP activity ( $\mu \mathrm{mol} \mathrm{Fe}(\mathrm{II}) / \mathrm{g}$ sample) by calibration with ferrous sulfate from $0-30 \mu \mathrm{M}$.

The cyto-protective activity of EPS fractions against oxidative cell damage was tested in rat pheochromocytoma PC12 cell culture, subjected to peroxide $\mathrm{H}_{2} \mathrm{O}_{2}$ treatment $[11,12]$. The EPS samples were pre-dissolved in phosphate buffered saline (PBS) at $10 \mathrm{mg} / \mathrm{L}$. The PC12 cell culture was maintained in RPMI 1640 medium supplemented with $10 \%$ fetal bovine serum in a $\mathrm{CO}_{2}$ incubator at $37{ }^{\circ} \mathrm{C}$. The activity test was performed on a 96 well-plate by treating the cells with $80 \mu \mathrm{M} \mathrm{H}_{2} \mathrm{O}_{2}$ and EPS sample solution at selected concentrations $(0.001-200 \mu \mathrm{g} / \mathrm{mL})$. The cell viability was measured by the MTT assay and represented in percentage relative to the native culture $(\mathrm{N})$ to culture without any treatment. 


\section{Conclusions}

An antioxidative glycopeptide Cs-GP1 has been isolated from the low $M \mathrm{w}$ fraction of EPS produced and released by the Cs-HK1 fungus into the liquid medium. Its molecular composition and structure have been partially characterized including the monosaccharide, amino acid composition, and the peptide chain sequence through hydrolysis and analytical experiments, though its glyco-chain structure remains unknown. Another distinct feature of Cs-GP1 is its presence as an extracellular product which is favorable for mass production by liquid fermentation and efficient recovery from the liquid medium. In other words, the present study has demonstrated the application of a medicinal fungus as the source or producer of novel bioactive glycopeptides.

\section{Supplementary Materials}

Supplementary Figures can be found at http://www.mdpi.com/1422-0067/15/10/17318/s1.

\section{Acknowledgments}

This work was supported by the Hong Kong Scholar Program jointly funded by the Chinese Government (XJ201115) and The Hong Kong Polytechnic University, and by grants from the Hong Kong Government UGC (GRF Projects PolyU5036/10P and PolyU 5033/11P).

\section{Author Contributions}

Jian-Yong $\mathrm{Wu}$ supervised the whole project and revised the manuscript; Xia Chen and Ka-Chai Siu contributed to the experiments and manuscript draft. All authors read the manuscript before submission.

\section{Conflicts of Interest}

The authors declare no conflict of interest.

\section{References}

1. Ooi, V.E.; Liu, F. Immunomodulation and anti-cancer activity of polysaccharide-protein complexes. Curr. Med. Chem. 2000, 7, 715-729.

2. Xu, X.; Yan, H.; Chen, J.; Zhang, X. Bioactive proteins from mushrooms. Biotechnol. Adv. 2011, 29, 667-674.

3. Samaranayaka, A.G.; Li-Chan, E.C. Food-derived peptidic antioxidants: A review of their production, assessment, and potential applications. J. Funct. Foods 2011, 3, 229-254.

4. Li, S.P.; Zhao, K.J.; Ji, Z.N.; Song, Z.H.; Dong, T.T.X.; Lo, C.K.; Cheung, J.K.H.; Zhu, S.Q.; Tsim, K.W.K. A polysaccharide isolated from Cordyceps sinensis, a traditional Chinese medicine, protects PC12 cells against hydrogen peroxide-induced injury. Life Sci. 2003, 73, 2503-2513.

5. Zhu, J.S.; Halpern, G.M.; Jones, K. The scientific rediscovery of a precious ancient Chinese herbal regimen: Cordyceps sinensis_-Part II. J. Altern. Complement. Med. 1998, 4, 429-457.

6. Paterson, R.R.M. Cordyceps: A traditional Chinese medicine and another fungal therapeutic biofactory? Phytochemistry 2008, 7, 1469-1495. 
7. Wong, J.H.; Ng, T.B.; Wang, H.; Sze, S.C.W.; Zhang, K.Y.; Li, Q.; Lu, X. Cordymin, an antifungal peptide from the medicinal fungus Cordyceps militaris. Phytomedicine 2011, 18, 387-392.

8. Wang, J.; Liu, Y.-M.; Cao, W.; Yao, K.-W.; Liu, Z.-Q.; Guo, J.-Y. Anti-inflammation and antioxidant effect of Cordymin, a peptide purified from the medicinal mushroom Cordyceps sinensis, in middle cerebral artery occlusion-induced focal cerebral ischemia in rats. Met. Brain Dis. 2012, 27, 159-165.

9. Leung, P.H.; Zhang, Q.X.; Wu, J.Y. Mycelium cultivation, chemical composition and antitumour activity of a Tolypocladium sp. fungus isolated from wild Cordyceps sinensis. J. Appl. Microbiol. 2006, 101, 275-283.

10. Leung, P.H.; Zhao, S.; Ho, K.P.; Wu, J.Y. Chemical properties and antioxidant activity of exopolysaccharides from mycelial culture of Cordyceps sinensis fungus Cs-HK1. Food Chem. 2009, 114, 1251-1256.

11. Huang, Q.L.; Siu, K.C.; Wang, W.Q.; Cheung, Y.C.; Wu, J.Y.; Huang, Q.-L.; Siu, K.-C.; Wang, W.-Q.; Cheung, Y.-C.; Wu, J.-Y. Fractionation, characterization and antioxidant activity of exopolysaccharides from fermentation broth of a Cordyceps sinensis fungus. Process Biochem. 2013, 48, 380-386.

12. Chen, S.; Siu, K.-C.; Wang, W.-Q.; Liu, X.-X.; Wu, J.-Y. Structure and antioxidant activity of a novel poly-N-acetylhexosamine produced by a medicinal fungus. Carbohydr. Polym. 2013, 3, 332-338.

13. Fabian, H.; Mäntele, W. Infrared spectroscopy of proteins. Handbook of Vibrational Spectroscopy; John Wiley \& Sons, Ltd.: Chichester, NJ, USA, 2002; Volume 5, pp. 3399-3425.

14. Barth, A. Infrared spectroscopy of proteins. Biochim. Biophys. Acta BBA-Bioenerg. 2007, 1767, 1073-1101.

15. Chen, S.; Xu, J.; Xue, C.; Dong, P.; Sheng, W.; Yu, G.; Chai, W. Sequence determination of a non-sulfated glycosaminoglycan-like polysaccharide from melanin-free ink of the squid Ommastrephes bartrami by negative-ion electrospray tandem mass spectrometry and NMR spectroscopy. Glycoconj. J. 2008, 25, 481-492.

16. Chen, S.; Li, G.; Wu, N.; Guo, X.; Liao, N.; Ye, X.; Liu, D.; Xue, C.; Chai, W. Sulfation pattern of the fucose branch is important for the anticoagulant and antithrombotic activities of fucosylated chondroitin sulfates. Biochim. Biophys. Acta BBA-Gen. Subj. 2013, 1830, 3054-3066.

17. Helenius, A.; Aebi, M. Intracellular functions of N-linked glycans. Science 2001, 291, 2364-2369.

18. Petrescu, A.J.; Wormald, M.R.; Dwek, R.A. Structural aspects of glycomes with a focus on $N$-glycosylation and glycoprotein folding. Curr. Opin. Struct. Biol. 2006, 16, 600-607.

19. Varki, A. Biological roles of oligosaccharides: All of the theories are correct. Glycobiology 1993, 3, 97-130.

20. Dwek, R.A. Glycobiology: Toward understanding the function of sugars. Chem. Rev. 1996, 96, 683-720.

21. Dwek, R.A.; Butters, T.D.; Platt, F.M.; Zitzmann, N. Targeting glycosylation as a therapeutic approach. Nat. Rev. Drug Discov. 2002, 1, 65-75.

22. Haltiwanger, R.S.; Lowe, J.B. Role of glycosylation in development. Annu. Rev. Biochem. 2004, 73, 491-537. 
23. Dube, D.H.; Bertozzi, C.R. Glycans in cancer and inflammation-Potential for therapeutics and diagnostics. Nat. Rev. Drug Discov. 2005, 4, 477-488.

24. Chen, H.M.; Muramoto, K.; Yamauchi, F. Structural analysis of antioxidative peptides from soybean $\beta$-conglycinin. J. Agric. Food Chem. 1995, 43, 574-578.

25. Mendis, E.; Rajapakse, N.; Byun, H.G.; Kim, S.K. Investigation of jumbo squid (Dosidicus gigas) skin gelatin peptides for their in vitro antioxidant effects. Life Sci. 2005, 77, 2166-2178.

26. Lowry, O.H.; Rosebrough, N.J.; Farr, A.L.; Randall, R.J. Protein measurement with the Folin phenol reagent. J. Biol. Chem. 1951, 193, 265-275.

27. Shevchenko, A.; Tomas, H.; Havlis, J.; Olsen, J.V.; Mann, M. In-gel digestion for mass spectrometric characterization of proteins and proteomes. Nat. Protoc. 2007, 1, 2856-2860.

28. Re, R.; Pellegrini, N.; Proteggente, A.; Pannala, A.; Yang, M.; Rice-Evans, C. Antioxidant activity applying an improved ABTS radical cation decolorization assay. Free Radic. Biol. Med. 1999, 26, 1231-1237.

29. Benzie, I.F.F.; Strain, J.J. The ferric reducing ability of plasma (FRAP) as a measure of antioxidant power by the FRAP assay. Anal. Biochem. 1996, 239, 70-76.

(C) 2014 by the authors; licensee MDPI, Basel, Switzerland. This article is an open access article distributed under the terms and conditions of the Creative Commons Attribution license (http://creativecommons.org/licenses/by/4.0/). 